\title{
Fasting Can Heal the Human Organism of Disease and Reverse the Ageing Process
}

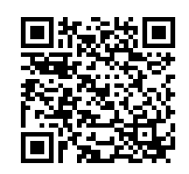

\author{
Michele d Moore* \\ University of Natural Health, United States
}

Submission: November 07, 2019; Published: December 13, 2019

*Corresponding author: Michele d Moore, University of Natural Health, United States

\begin{abstract}
Fasting is often thought of as starvation and many find it hard to grasp the thought of going without food for any period of time, even though the abstinence from food can have a profound effect on the body. During this house cleaning process where the body is able to relieve itself of the task of digesting food and eliminating waste, it can concentrate more of its energy on repairing and healing the body. If the fast is long enough, it has the power to heal the body of disease and reverse the ageing process.
\end{abstract}

\section{Introduction}

A proper fast requires mental awareness that the person will abstain from all foods and drinks except water for a fixed period of time or until the natural return of hunger, which is a throat and mouth sensation, similar to the feeling of thirst. Starvation is the depravation and denying the body of food, whereas fasting is a conscious decision made for the purpose of giving the digestive tract a physiological vacation and reaping the benefits that derive from this process. At this time of physiological rest, it is also advised that the body gets physical and mental rest to assist in the full process of rest and recuperation. Every task that the body performs requires expenditure of nerve energy; digestion, exercise, working and studying all use up bodily resources and when these are ceased, they body has the opportunity to focus on other areas that often get neglected due to our busy and hectic lifestyles. When food is consumed, extra blood is required for the process of digestion and as the body can only attend to one task at a time, it often has to divert its attention to the process of digestion. In many cases when one exercises immediately after eating, the process of digestion is neglected to focus on the main task at hand of providing extra blood to the vital organs during the exercise. This prohibits digestion or the food is simply left to ferment causing indigestion and if continued over a period of time, may lead to more serious symptoms or disease.

During a fast, any nutritive material that remains in the digestive tract as well as adipose tissue and glycogen are utilised first. Once these reserves are depleted, non-essential tissues are used for the preservation and ability of the vital organs to functioning effectively, after which the body begins to break down dead or diseased tissue during a process called autolysis. The word autolysis is used in physiology to describe the process of digestion or disintegration of tissue by ferments generated in the cells - a self-digestion or intracellular digestion process. It is during this process that diseased tissues are broken down, dead cells and tumour-like growths are absorbed and used or eliminated. The various kinds of tumours that exist are made up of bone, tissue and flesh. A lump in the breast, a neoplasm (new growth) may be nothing more than an enlarged lymphatic or mammary gland. Fasting is the most profound method possible to facilitate the autolysis of a tumour whether benign or malignant and it is a safe physiological process that presents no danger to the body. "I have seen repeated instances of the absorption of tumours in my own patients. I had one complete recovery in the case of a uterine cancer during a thirty days' fast. I have seen numerous small tumours completely absorbed and large ones greatly reduced in size" [1]. Tissues and organs are repaired more rapidly during a fast than while eating nourishing food. As the body undergoes a complete internal renovation, new structures are built, and the redistribution of nutritive material is also seen during a fast. There are many other organic changes that occur during a fast that reverse the ageing of the body by rejuvenating the cells.

The skin for example undergoes revitalization that is evident in the disappearance of blotches, blemishes and fine lines as well as improved circulation. One looks youthful and younger with brighter eyes and improved skin tone. The improvement of memory and clearness of the mind after a fast, provides the brain with a 
cleaner blood stream to think and perform other mental tasks due to the elimination of the toxins that previously loaded the brain. Whether you are old or young, the ageing process is reversed by fasting giving you a renewed standard of health with your whole organism and functions improved. "The daily renewal of its cells and tissues prevents old age and early death for considerable time, despite the worst abuses which are heaped upon the bodies of most of us. Fasting enables the processes of renewal to out-distance the processes of degeneration and the result is a higher standard of health" [2].

"A great change in cell life and structure takes place during the fast and it is well to continue the fast until this change is complete and nothing, but healthy tissue remains. In this way a new body emerges from the process. It is thin, but ready to be rebuilt upon normal lines. After such an overhauling process, when the body has been largely torn down and thrown away, when the accumulated waste and debris of a life-time have been refined or cast out and, after the chemical readjustment, occasioned by the fast, has occurred, the body that is properly cared for is built anew and its youth renewed"[3]. We natural hygienists do not believe in 'cures' for the body and although fasting is not a cure, it provides the favorable conditions for the body to execute its own healing activities, repair any damage and produce a healthy internal environment for the living organism to thrive. The abstinence of food affords the organs of the body a much needed rest through the cessation of decomposing poison in the alimentary canal, allowing the body to recuperate and repair its organs, remove circulating and deposited toxins, normalize blood chemistry, rejuvenate cells and tissue, absorb effusions and growths and improve the body's powers of digestion and assimilation.

\section{References}

1. Herbert M Shelton (1950) The Hygienic System Vol II Fasting and Sunbathing, Health Research Books. Pomeroy p. 43.

2. Ibid, 86 .

3. Ibid, 88 